\title{
Single Organic Droplet Collision Voltammogram via Electron Transfer Coupled Ion Transfer
}

\author{
Cheng Liu, ${ }^{\dagger, \text { Il }}$ Pekka Peljo, ${ }^{\ddagger}$, Iङ Xinjian Huang, ${ }^{\dagger}$ Wenxue Cheng, ${ }^{\dagger}$ Lishi Wang, ${ }^{*}{ }^{\dagger} \odot$ and Haiqiang Deng ${ }^{*}, \S$ \\ ${ }^{\dagger}$ School of Chemistry and Chemical Engineering, South China University of Technology, Guangzhou 510641, China \\ ${ }^{\ddagger}$ Laboratoire d’Electrochimie Physique et Analytique, École Polytechnique Fédérale de Lausanne (EPFL Valais Wallis), Rue de \\ I'Industrie, 17, 1951 Sion, Switzerland \\ ${ }^{\S}$ Institute of Chemistry, The Hebrew University of Jerusalem, Jerusalem 9190401, Israel
}

\section{Supporting Information}

ABSTRACT: Single-emulsion toluene oil droplets (femtoliter) containing a hydrophobic redox probe that are dispersed in water stochastically collide with an ultramicroelectrode (UME). The fast-scan cyclic voltammetry (FSCV) or Fouriertransformed sinusoidal voltammetry (FTSV) is applied: the UME was scanned with a fast, repetitive triangular, or sinusoidal potential, and its current in time/frequency domains were monitored. The electron transfer at the UME/oil interface is coupled with ion transfer at the oil/water interface. Thus, the obtained transient voltammograms of a myriad of ions were used to estimate thermodynamics of ion transfer at the toluene/ water interface. Additionally, the single-droplet voltammogram combined with finite element simulations reveal the droplet's size and shape distributions. Four collision mechanisms with new physical insights were also uncovered via comprehensive analysis of phase angle in the frequency domain, time domain FSCVs, and finite element simulations.

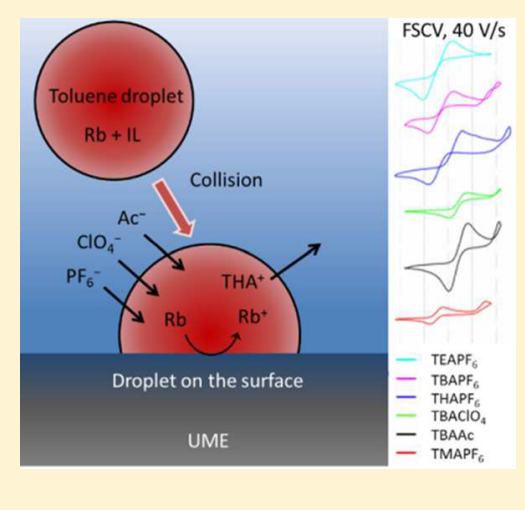

I on translocation across the biomembrane is a ubiquitous process that plays an essential role in biology. An oil/water $(\mathrm{o} / \mathrm{w})$ interface or the interface between two immiscible electrolyte solutions (ITIES) is such a biomimetic interface. ${ }^{1}$ The ion transfer (IT) at the ITIES underpins a myriad of biological (e.g., proton pump ${ }^{2}$ ) and industrial processes (e.g., phase transfer catalysis ${ }^{3}$ and hydrometallurgy ${ }^{4,5}$ ). Until now, IT at an externally polarizable ITIES as a function of Galvani potential difference (applied at the ITIES) $)^{6-10}$ was studied by employing the four-electrode setup ${ }^{11-13}$ and droplet-modified three-electrode system $^{14-17}$ for macro-ITIES; micro/nanopipettes $^{18-24}$ and/or holes, ${ }^{25}$ and their arrays, ${ }^{26}$ for microand nano-ITIES; and scanning electrochemical microscopy (SECM). ${ }^{27-31}$ Recently, we developed a novel method to study ion transfer across the ITIES at the level of a single femtoliter oil droplet upon its stochastic collision with an ultramicroelectrode (UME). ${ }^{32}$ An extremely lipophilic redox probe-rubrene $(\mathrm{Rb})$ is trapped within an emulsion oil droplet. By applying a sufficiently and constantly positive potential on the UME, upon collision of the oil droplet on the UME, electron transfer (ET) at the ultramicroelectrode/oil (UME/o) interface will couple the IT at the o/w interface to maintain the electroneutrality in the droplet. This process is monitored as a function of time (i.e., chronoamperometry). This approach is considered as a type of single-particle collision electrochemistry, ${ }^{33-42}$ which can be further classified into four major categories: current blockage of existing redox reaction, ${ }^{43,44}$ bulk electrolysis, ${ }^{32,45}$ electrocatalytic amplification, $^{46-51}$ and charge displacement. ${ }^{52}$ Single-particle collision events normally last for a short period of time; therefore, the majority of the literature in this field employs chronoamperometry as the signal readout method. ${ }^{35}$ A limitation of chronoamperometry is that only transient current response is obtained without chemical/potential resolution. Fortunately, fast-scan cyclic voltammetry (FSCV) ${ }^{37,50,53}$ and high-frequency Fourier-transformed sinusoidal voltammetry (FTSV) $)^{51,54}$ have recently been applied to single-particle collision electrochemistry to provide chemical/potential resolution. The operating principles of FSCV and FTSV have been detailed previously. ${ }^{50,51,55,56}$ Note that Compton and co-workers employed conventional CV (10 and $100 \mathrm{mV} \mathrm{s}^{-1}$ ) rather than FSCV to record transient electrochemistry of emulsion droplets containing Nile Red as the photo/redox probe ${ }^{57}$ and of individual palladium-coated carbon nanotubes using hydrogen oxidation as the indicator reaction ( $\mathrm{CV}$ in $\left.50 \mathrm{mV} \mathrm{s}^{-1}\right){ }^{58}$ So a full voltammogram of a single particle has not yet been achieved in their studies.

Herein, we establish a novel approach that combines the advantages between the methodology of studying IT at the single-emulsion droplet, $^{32}$ FSCV/FTSV, ${ }^{50,51}$ and numerical finite element simulations via COMSOL Multiphysics to gain new physical insights into this intriguing ET coupled IT process. Briefly, a variety of ions at the same concentration initially located either inside the aqueous or oil phase have been

Received: May 31, 2017

Accepted: July 31, 2017

Published: July 31, 2017 

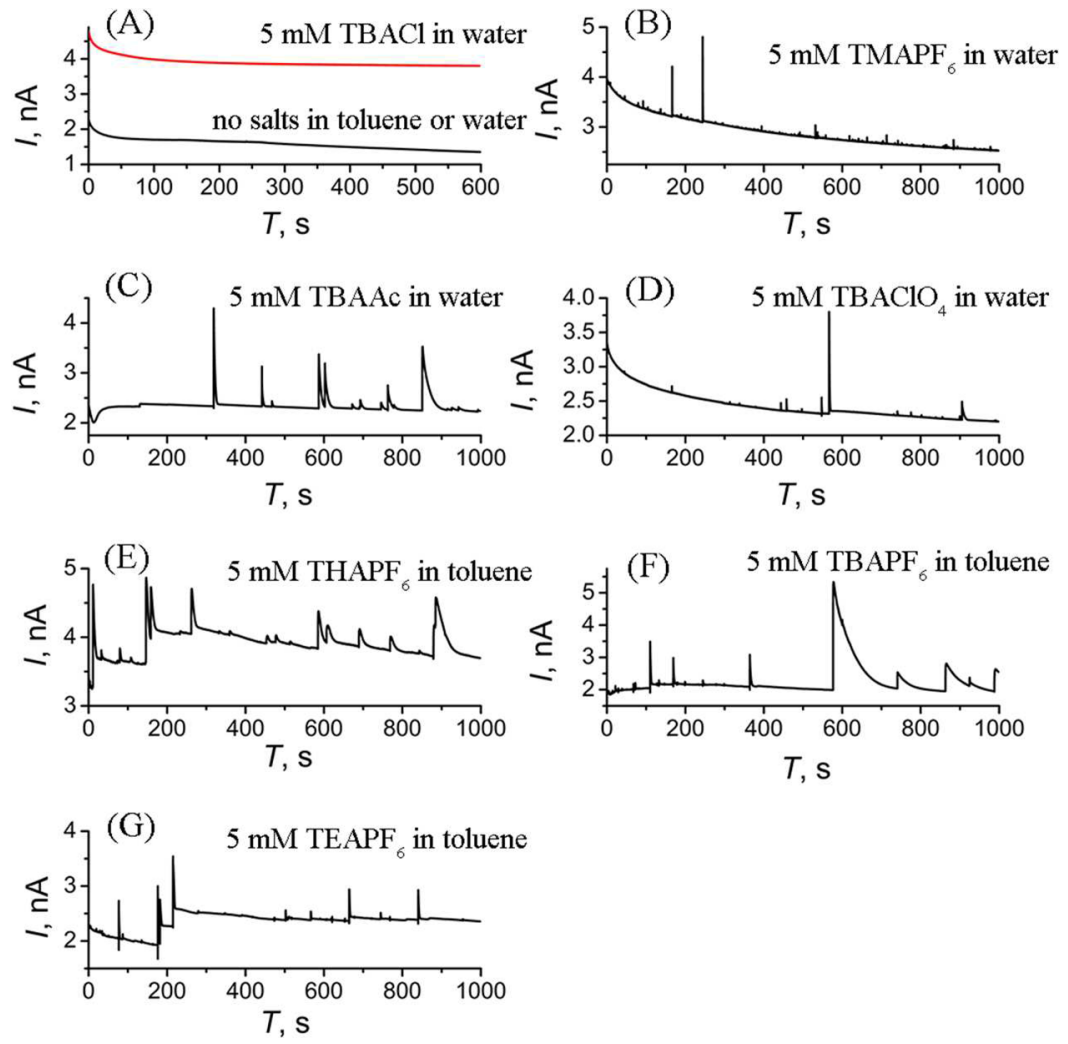

Figure 1. Fundamental harmonic current component obtained by FSCV at a carbon fiber UME (C UME, diameter $=10 \mu \mathrm{m})$ with toluene emulsion droplets ( $5 \mathrm{mM}$ rubrene+400 mM IL-PA) in $36.7 \mathrm{pM}$. The parameters of all the above experiments were as follows: bias potential $0.8 \mathrm{~V}$ vs. a Ag/ $\mathrm{AgCl}$ wire quasireference electrode (QRE), peak amplitude $500 \mathrm{mV}$, frequency $20 \mathrm{~Hz}$ (viz. $40 \mathrm{~V} / \mathrm{s}$ ), quiet time $2 \mathrm{~s}$. (A) No salt was dissolved in both toluene and aqueous phases (black line) and $5 \mathrm{mM} \mathrm{TBACl}$ was dissolved in the aqueous phase (red line). (B), (C) and (D) $5 \mathrm{mM} \mathrm{TMAPF}, 5 \mathrm{mM}$ TBAAc, or $5 \mathrm{mM} \mathrm{TBAClO}_{4}$ was dissolved in the aqueous phase, respectively. (E),(F),(G) $5 \mathrm{mM} \mathrm{THAPF}_{6}, 5 \mathrm{mM} \mathrm{TBAPF}$, or 5 mM TEAPF 6 was dissolved in the toluene phase, respectively. The acronyms of these salts were explained in the Experimental Section.

systematically studied, as well as a specific ion at different concentrations in the aqueous phase. The high symmetry of the FSCV peak currents confirms that the rubrene radical cation $\left(\mathrm{Rb}^{+}\right)$produced in the ET reaction is strictly confined within the oil droplet (when transferrable ions are present in either oil or water phase). This corroborates prior results. ${ }^{32} \mathrm{We}$ also estimated the apparent transfer energies and the formal ion transfer potentials of a myriad of ions at the toluene/water interface with the linear relationship of the Gibbs transfer energies of ions at the toluene/water interface and those at the 1,2-dichloroethane (DCE)/water interface. ${ }^{59}$ Comparison between experimental FSCVs of the ET coupled IT processes and the simulated ones via COMSOL Multiphysics software has shed new light on the electrochemical reaction and collision mechanisms. For example, droplet size and shape distributions and new collision mechanisms were revealed by the quantitative and high-temporal-resolution electrochemical method developed herein. The developed method is termed as "Single Organic Droplet Collision Voltammogram via Electron Transfer Coupled Ion Transfer". Besides the proof-of-concept shown herein, utilizing ITIES electrochemistry with low-dielectricconstant organic phases, this method may also be applied in electrochemistry in low-dielectric-constant media at the singleemulsion droplet level, nanoscience, ${ }^{39}$ electrocatalysis, ${ }^{34}$ exocytosis, ${ }^{60,61}$ vesicles/liposomes, ${ }^{62,63}$ and so forth.

\section{EXPERIMENTAL SECTION}

Reagents and Materials. All reagents were used as received without further purification unless otherwise mentioned. Rubrene ( $\mathrm{Rb}, 98.5 \%)$, tetrabutylammonium chloride (TBACl, 98\%), tetrabutylammonium perchlorate $\left(\mathrm{TBAClO}_{4}\right.$, $98 \%$ ), tetramethylammonium hexafluorophosphate (TMAPF${ }_{6}$, $99 \%)$, tetrabutylammonium hexafluorophosphate TBAPF $_{6}$, $99 \%)$, tetraethylammonium hexafluorophosphate (TEAPF 6 , 99\%), and hydroxymethylferrocene (FcMeOH, 97\%) were purchased from J\&K. Tetrahexylammonium hexafluorophosphate $\left(\mathrm{THAPF}_{6}, 97 \%\right)$, tetrabutylammonium acetate (TBAAc, $97 \%)$, and trihexyltetradecylphosphonium bis(trifluoromethylsulfonyl)amide (IL-PA, also noted as $\mathrm{P}_{66614} \mathrm{NTf}_{2}{ }^{21}$ see Table S1 in Supporting Information (SI), $\geq 97 \%$ ) were purchased from Sigma-Aldrich, Energy Chemicals and Strem Chemicals, respectively. Ferrocene (Fc, $\geq 99 \%$ ), $\mathrm{KNO}_{3}$ (analytical grade) and $\mathrm{NaOH}$ (analytical grade) were obtained from Tianjin Fuchen Chemical Reagent Factory. Toluene, isopropyl alcohol, absolute ethanol, and acetone were obtained from Tianjin Fuyu Fine Chemical and all are of analytical grade. The $10 \mu \mathrm{m}$ platinum ultramicroelectrode (Pt $\mathrm{UME}), \mathrm{Ag} / \mathrm{AgCl} /$ saturated $\mathrm{KCl}$ reference electrode and platinum wire counter electrode were purchased from Shanghai Xianren Instrument, while the $10 \mu \mathrm{m}$ carbon fiber ultramicroelectrode (C UME) was fabricated following the previous report. ${ }^{64}$ All the aqueous solutions were prepared from the Millipore water $(\geq 18.2 \mathrm{M} \Omega \cdot \mathrm{cm})$. 
Instrumentation. Fast-scan voltammetry experiments were performed with a home-built instrument. Details about this instrument can be found in our prior work. ${ }^{51}$ Note that this instrument can generate not only sinusoidal but also triangular potential waveforms.

All the electrochemical measurements were undertaken in a one-compartment three-electrode glass cell housed in a Faraday cage. A Pt wire served as the counter electrode, with an $\mathrm{Ag}$ / $\mathrm{AgCl} /$ saturated $\mathrm{KCl}$ reference electrode or an $\mathrm{Ag} / \mathrm{AgCl}$ wire quasi-reference electrode, respectively. Before each electrochemical measurement, the Pt UME or C UME was polished with polishing films $(0.05 \mu \mathrm{m}$, NTT-ATADS, Japan), which have been widely used for optical fiber polishing. Then Pt UME and $\mathrm{C}$ UME were cycled from -0.215 to $1 \mathrm{~V}$ and -1 to $1 \mathrm{~V}$ vs $\mathrm{Ag} / \mathrm{AgCl} /$ saturated $\mathrm{KCl}(0.1 \mathrm{~V} / \mathrm{s})$ in $0.5 \mathrm{M} \mathrm{H}_{2} \mathrm{SO}_{4}$ under argon, respectively. The cleanliness of UME was checked by an optical microscope with $400 \times$ magnification. After experiments, the tips of $\mathrm{Pt}$ and $\mathrm{C}$ UMEs were first rinsed with acetone and isopropyl alcohol, respectively, and then rinsed with copious Millipore water to clean the electrode surface prior to storage. For preparation of emulsion, a JY92-2D ultrasonic processor (Xinzhi, Ningbo, China) with a microtip probe was employed.

Preparation of the Emulsions. The procedure of emulsion preparation followed the published literature. ${ }^{32}$ Briefly, $5 \mathrm{mM}$ rubrene and $400 \mathrm{mM} \mathrm{IL}-\mathrm{PA}$ were dissolved in $5 \mathrm{~mL}$ of toluene. Then $0.1 \mathrm{~mL}$ of toluene (rubrene+IL-PA) was mixed with $5 \mathrm{~mL}$ of water, followed by vigorous vortexing. After that, an ultrasonicator (500 W, amplitude 40\%) was applied immediately using the pulse mode ( $7 \mathrm{~s}$ on, $3 \mathrm{~s}$ off, 26 cycles repeated). A variety of salts were dissolved in either the toluene phase or the aqueous phase to investigate either the cation transfer or anion transfer across the o/w interface, respectively. The preparation of rubrene-encapsulated oil in water emulsion and the following electrochemical collision measurements should be carried out under dark to avoid rubrene photo-oxidation. ${ }^{65}$ The droplet dispersion showed a bimodal size distribution, as determined by dynamic light scattering (DLS, see Figure S1 in SI), and this distribution was found to depend on both time and salt composition (Figure S4 in SI).

\section{RESULTS AND DISCUSSION}

Fundamental Harmonic Current Magnitude Obtained by FSCV. Figure 1 illustrates the fundamental harmonic current magnitude of FSCV recorded for collision experiments employing the emulsion of toluene encapsulating $\mathrm{Rb}$ and ionic liquid, that is, IL-PA (Section 1 in SI), dispersed in water, with and without additional ions in the oil or aqueous phase. Due to the extremely hydrophobic structure of rubrene (see the SI in ref 32), no spikes were seen in the current response (Figure 1A, black line) even after applying potential up to $1.3 \mathrm{~V}$. The impurities in the commercial IL-PA were not observable in the experimental results. A similar result was obtained with addition of $5 \mathrm{mM} \mathrm{TBACl}$ in aqueous phase (Figure 1A, red line), indicating that the $\mathrm{Cl}^{-}$anion was too hydrophilic to transfer into toluene phase. It also implies that the trace $\mathrm{Cl}^{-}$dissolving from the $\mathrm{Ag} / \mathrm{AgCl}$ wire will not affect the results during all the experiments. According to this observation, and our previous investigation that transfer energy of rubrene cation is higher than that for decamethylferrocenyl or 1,1',3,3'-tetra(2-methyl2-nonyl)ferrocenyl cations, ${ }^{32}$ rubrene cation is expected to transfer at similar or lower potentials than chloride. When other less hydrophobic or hydrophilic ions were dissolved in either the toluene droplets or the continuous aqueous phase, respectively, clear current spikes were observed marking the event of "ET coupled IT" during single-emulsion droplet collision electrochemical measurements (Figure $1 \mathrm{~B}-\mathrm{G}$ ). ${ }^{32}$ This implies that the transfer of other ions, rather than $\mathrm{Rb}^{+}$or the cation of IL-PA, ${ }^{21}$ across the $\mathrm{o} / \mathrm{w}$ boundary facilitates redox reaction of rubrene at $\mathrm{UME} / \mathrm{o}$ interface. Note that some current signals last a long period of time in the late stage in Figure 1C,E,F. This observation could be due to the ET coupled IT of coalesced (i.e., bigger) droplets colliding on the microelectrode. It is seen clearly in Figures S4 and S5 in SI that the emulsion droplets can grow larger at longer time scales. These ions have intermediate lipophilicity/hydrophilicity so that they can cross the $\mathrm{o} / \mathrm{w}$ interface with a relatively low ion transfer potential within the obtainable potential window. However, the hydrophilicity and lipophilicity of these different ions and the mechanism of the droplet collisions remain unclear. This can be evaluated by the time-domain voltammetric analysis of FSCV and finite element simulations.

Time-Domain Background-Subtracted FSCV. The application of FSCV allows acquisition of CV for each singlecollision event by means of background subtraction which is illustrated in Figure 2A. Briefly, in panel "I" of Figure 2A, an abrupt current signal (533.7 to $535 \mathrm{~s}$ ) attributed to a singleemulsion droplet collision event is superimposed on the background current (532 to $533.5 \mathrm{~s}$ ). Then the current from 532 to $533.5 \mathrm{~s}$ is taken as the background and can be subtracted to get the net collision signal (panel "II" in Figure 2A). Accordingly, a recovered time-domain $\mathrm{CV}$ of the collision event starting at $533.7 \mathrm{~s}$ is obtained and shown in panel "III" of Figure 2A. Using this approach, CVs and peak/half-wave potentials of some ions transfer were recorded and shown in Figures $2 \mathrm{~B}$ and $2 \mathrm{C}$, respectively. It should be stressed that the CVs in Figure 2B are only exemplary, and $\mathrm{CV}$ characteristics vary with the size, collision contact angle and/or collision time of toluene droplets (cf. Figures S3-S5 in SI).

Ion Distributions and Thermodynamic Analysis. The initial distribution of ions between aqueous and organic phases can be calculated as detailed in Section 2 in the SI, showing that all the salts except $\mathrm{THAPF}_{6}$ will primarily reside in the aqueous phase. However, the low solubility of some of the salts like $\mathrm{TEAPF}_{6}$ and $\mathrm{TBAPF}_{6}$ in the aqueous phase, as well as the presence of $400 \mathrm{mM}$ of the surface-active IL-PA in the toluene phase might complicate the issue.

From the thermodynamic calculations, only the transfer of $\mathrm{PF}_{6}^{-}, \mathrm{THA}^{+}, \mathrm{ClO}_{4}^{-}$, and $\mathrm{Ac}^{-}$can take place, as $\mathrm{PF}_{6}^{-}$salts with $\mathrm{TMA}^{+}, \mathrm{TEA}^{+}$and $\mathrm{TBA}^{+}$cations are preferably distributed in the aqueous phase (Tables $S 1$ and S2 in the SI). The half-wave potentials $E_{1 / 2}$ obtained with $\mathrm{TEAPF}_{6}, \mathrm{TBAPF}_{6}$, and $\mathrm{TMAPF}_{6}$ in the FSCV are rather similar (Figure $2 \mathrm{C}$ ), indicating that the signal is indeed due to transfer of $\mathrm{PF}_{6}^{-}$. However, the peak current distributions shown in Figure S3 in SI do not exactly follow the equilibrium concentrations in Table S2. For example, the $\mathrm{TMAPF}_{6}, \mathrm{TBAAc}$ and $\mathrm{TBAClO}_{4}$ should have 50 times higher concentration of the transferring anion than the solutions with $\mathrm{TEAPF}_{6}$ and $\mathrm{TBAPF}_{6}$, but currents of similar or lower magnitude are obtained (Figure S3 in SI). This indicates that the droplet size distribution is affected by salts, and this observation was confirmed by DLS studies (Figure S4 in SI). Finite element (FE) simulations via COMSOL (vide infra) show that a collision by a droplet with a diameter below 1 $\mu \mathrm{m}$ results in an FSCV peak current of less than $100 \mathrm{pA}$, and hence, small droplets are difficult to observe by electro- 


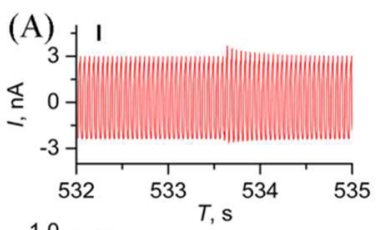

(B)
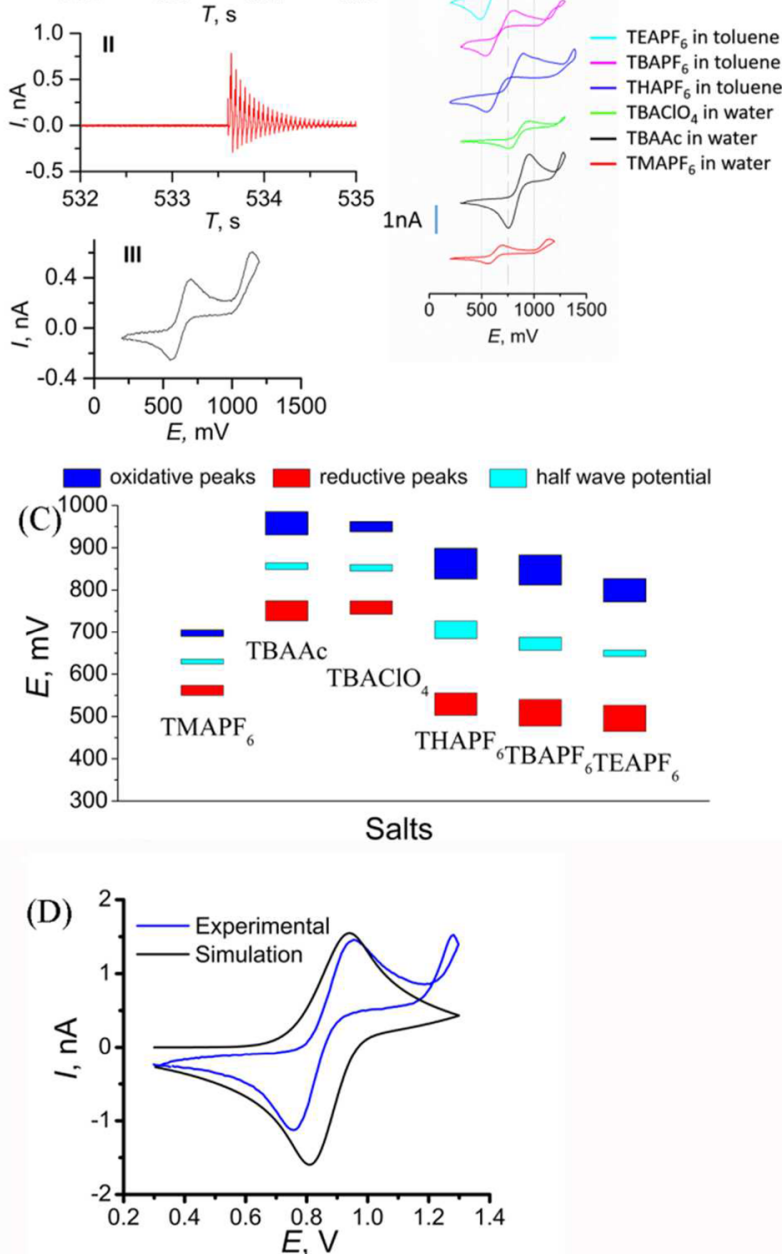

Figure 2. (A) Schematic depiction of the method for background subtraction in FSCV. (I) The raw current-time $(i-t)$ profile of an emulsion droplet collision signal obtained by FSCV. (II) The same $i-t$ profile after background (532 to $533.5 \mathrm{~s}$ ) subtraction. (III) Example of the recovered time-domain $\mathrm{CV}$ of a collision signal at the moment of $533.7 \mathrm{~s}$ after background subtraction. The frequency of the excited triangular potential wave was $20 \mathrm{~Hz}$. (B) Example of the recovered $\mathrm{CVs}$ of electron transfer coupled with different kinds of ions transfer during emulsion droplets colliding on the C UME recorded by FSCV. (C) The range (i.e., error) bar chart of peak and half-wave potentials of obtained collision signals from Figure 2B. (D) Comparison of the simulated CV (black curve) with the experimental one (blue curve, $\mathrm{Ac}^{-} /$TBAAc in panel B).

chemistry. The size distribution of larger droplets is qualitatively in accordance with the peak current distribution (Figures S3 and S4). Additionally, the partitioning of ions can be complicated by the surface-active IL-PA, as illustrated recently by interfacial dissociation of salts composed of a hydrophilic and a hydrophobic ion. ${ }^{66}$ The anion of the IL-PA has a higher Galvani transfer potential than that of $\mathrm{ClO}_{4}{ }^{-}, \mathrm{PF}_{6}{ }^{-}$, or $\mathrm{Ac}^{-}$(Table S1 in SI), ${ }^{21}$ so the IL-PA will not significantly affect the distribution of salts by its partition into the aqueous phase. It is seen from Table S1 that the lipophilicity of anions follows the order: $\mathrm{NTf}_{2}^{-}>\mathrm{PF}_{6}^{-}>\mathrm{ClO}_{4}^{-}>\mathrm{Ac}^{-}$. The experimental trend is in good agreement with that obtained at the DCE/w interface: ion transfer potential of $\mathrm{Ac}^{-}$is higher than $\mathrm{ClO}_{4}^{-}$and much higher than that of $\mathrm{PF}_{6}{ }^{-67}$ albeit there is a big difference in the ion solvation environment between DCE and toluene. ${ }^{68}$

Larger peak-to-peak separation observed with $\mathrm{TEAPF}_{6}$, $\mathrm{TBAPF}_{6}$, and $\mathrm{THAPF}_{6}$ is because of the ohmic voltage (iR) drop in the less conductive aqueous phase TEAPF $_{6}$ and TBAPF $_{6}$ are not very soluble in the aqueous phase, while THAPF $_{6}$ is present mostly in the organic phase). This effect is evidenced from Figure 2C, where the peak potential difference for all these cases is larger than that for other salts. Also, the $i R$ drop can change during the experiment as insertion of salts into the aqueous solution will dramatically increase the conductivity. $\mathrm{TEAPF}_{6}, \mathrm{TBAPF}_{6}$, and $\mathrm{THAPF}_{6}$ are only slightly soluble in aqueous phase, and thus, this effect will be small and is limited by the salt precipitation. On the contrary, the presence of a large amount of supporting electrolyte (i.e., IL-PA) in the toluene phase will not change the conductivity of the organic phase noticeably for salts of TMAPF 6 , TBAAc, and TBAClO The ratios between the oxidative and reductive peak currents for each salt were nearly equal to 1 (Table S3 in SI), indicating that the overall ET coupled IT process was reversible, and $\mathrm{Rb} /$ $\mathrm{Rb}^{+}$was encapsulated strictly in toluene droplets. In addition, the narrow range of potential distribution demonstrated in Figure $2 \mathrm{C}$ implied that the quasi-reference electrode of $\mathrm{Ag} /$ $\mathrm{AgCl}$ wire could be stable in both water and oil phases at least within the time scale $(1000 \mathrm{~s})$ of electrochemical collision measurements.

Finite Element Simulations. FE simulations (Section 5 in SI for further details) of the FSCV with $5 \mathrm{mM}$ TBAAc in the aqueous phase were done to understand better the ET-IT process. The droplet on the UME surface was approximated as a polar cap of a sphere with the same volume as the original spherical droplet. Both droplet radius $r_{\mathrm{d}}$ and the ratio of polar cap height $h$ to $r_{\mathrm{d}}$ were varied to obtain reasonable agreement with the experimental FSCV, with $r_{\mathrm{d}}=2 \mu \mathrm{m}$ and $h=1.4 r_{\mathrm{d}}$, as shown in Figure 2D. This experimental FSCV shows a collision with a rather large droplet, as the nominal currents for $5 \mathrm{mM}$ TBAAc are considerably lower (Figure S3B in SI), reflecting expected modal radius from the DLS measurements (Figure S1 in SI). However, the droplet will change its shape as a function of the electrode potential due to electrowetting onto the UME surface, ${ }^{51}$ making more accurate simulations very challenging. However, the simulation showed that $i R$ drop in the toluene solution is negligible and that both the entire UME surface in contact with the droplet and the droplet surface with aqueous phase are electrochemically active even though current density is higher at the three-phase boundary (Figure S8 in SI), which agrees partially with previous speculation. ${ }^{32}$

To investigate the effect of the droplet shape on the voltammetry, the cap height was varied while keeping the droplet radius constant at $2 \mu \mathrm{m}$. The results are shown in Figure 3. In these simulations, the diffusion coefficient of $\mathrm{Rb}$ in aqueous and toluene, $D_{\mathrm{aq}}$ and $D_{\text {org }}$ were taken as $2 \times 10^{-6} \mathrm{~cm}^{2}$ $\mathrm{s}^{-1}$ and $3 \times 10^{-6} \mathrm{~cm}^{2} \mathrm{~s}^{-1}$ respectively, ${ }^{32}$ while all the $k^{0}$ values were set as $0.01 \mathrm{~cm} \mathrm{~s}^{-1}$. Note that a smaller volume of the aqueous phase was used in the model to reduce the computational time to ca. $3 \mathrm{~h}$. The purpose of this strategy is to capture the voltammetric trend using the model, as in all cases the reaction is limited by diffusion of $\mathrm{Rb}$ inside the toluene droplets. Smaller values of $h$ result in a larger radius of the interface between toluene and underlying UME (i.e., larger 
(A)

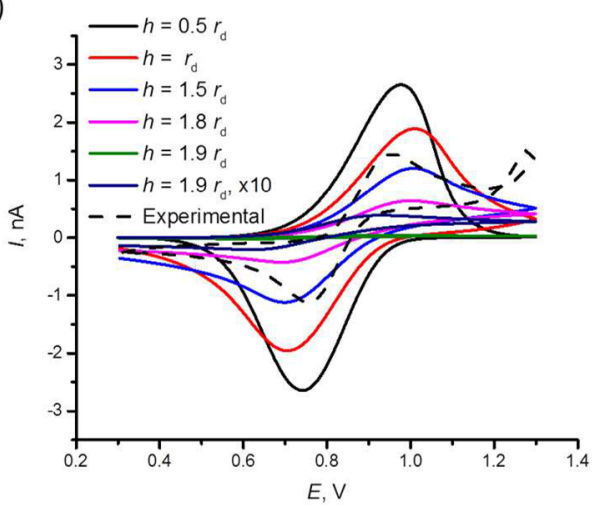

(B)

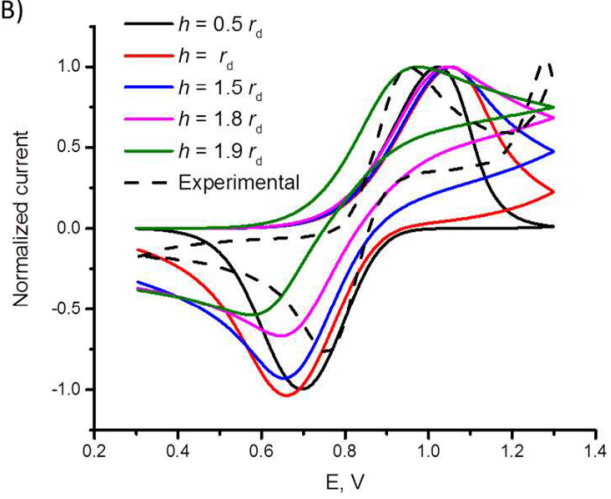

Figure 3. (A) Effect of the droplet cap height on the simulated FSCV. The current for the droplet $h=1.9 r_{\mathrm{d}}$ was multiplied by 10 to make the features visible (green vs. navy blue curve). $r_{\mathrm{d}}=2 \mu \mathrm{m}$ (B) FSCVs normalized by the oxidation peak current for better visualization and comparison.

$\left.r_{\mathrm{e}}\right)$. However, smaller values of $h$ are not reasonable as parts of the droplet will reside outside the active electrode surface (Note that UME radius is $5 \mu \mathrm{m}$ ). In this case both $\mathrm{o} / \mathrm{w}$ interface and UME/o interface have similar areas, and the electrochemical response resembles the thin-layer cell. ${ }^{69,70}$ The peak current decreases with increasing $h$, while the peak separation increases. The current decreases sharply with $h$ increasing from 1.8 to $1.9 r_{\mathrm{d}}$, because the available electrode area for the ET reaction decreases abruptly. For the very small UME/o interface with $h=1.9 r_{\mathrm{d}}$ the current needs to be multiplied by a factor of 10 for the features to be visible (green vs navy blue colored curves in Figure $3 \mathrm{~A}$ ). The FSCVs, normalized by the oxidative peak current (to better distinguish the trends), are shown in Figure 3B.

The effect of the droplet size was also investigated by FE simulations, with parameters described in Table S5 in SI, and the results are shown in Figure 4. As the droplet size gets smaller, the voltammetry starts to resemble the thin-layer cell, ${ }^{69,70}$ as diffusion of the redox mediator is confined to a smaller space.

The simulated voltammograms of different droplet sizes (Figure 4) show that smaller droplets should feature thin-layer type behavior, if the shape of the droplets remains unchanged. The comparison of the exemplary experimental voltammograms of different peak currents (Figure S5 in SI) with the simulated curves reveals that experimental curves show shapes typical for processes limited by linear diffusion. This indicates that the small currents likely result from an impact of relatively large droplet with high value of $h$ (i.e., $h \geq 1.9 r_{\mathrm{d}}$, refer to
(A)

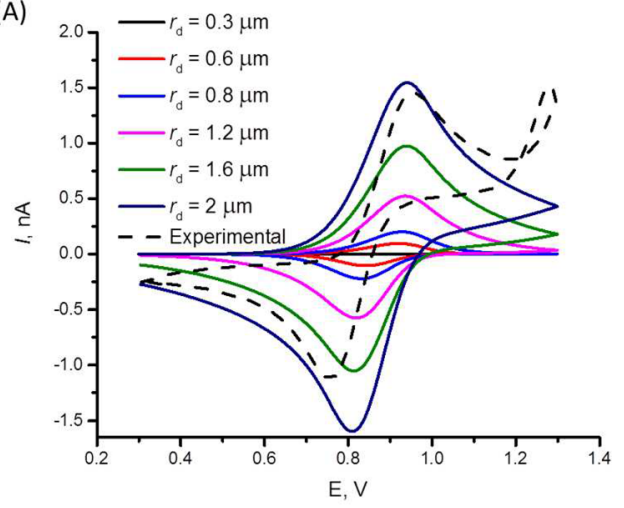

(B)

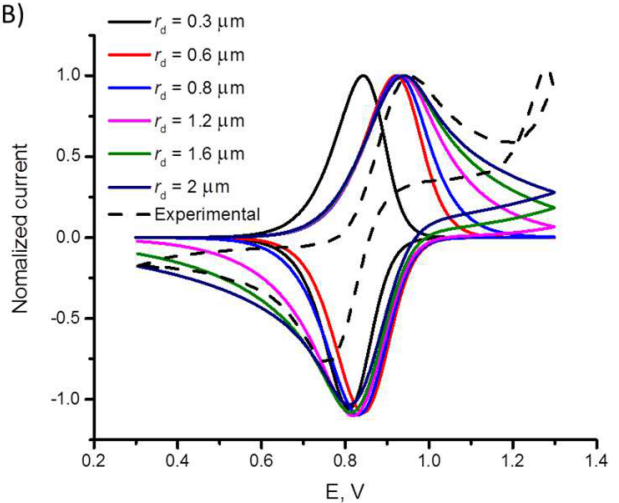

Figure 4. (A) Effect of the droplet size on the simulated FSCV, $h=1.4$ $r_{\mathrm{d}}$. (B) Simulated FSCVs normalized by the oxidation peak current.

Figures 3 and 4). Therefore, the peak current histograms (Figure S3 in SI) reflect not only the size distribution but also the shape distribution upon collision, and more detailed analysis is complicated by the time-dependent size distribution (Figure S4 in SI). Qualitative agreement between experiment and simulation is obtained with $r_{\mathrm{d}}=2 \mu \mathrm{m}$ and $h=1.4 r_{\mathrm{d}}$ as shown in Figure 2D. Note that the experimental reduction peak current is lower with respect to the simulated one, and the experimental reduction peak potential is shifted negatively. Better fit between experiments and simulations could be obtained by increasing both the droplet radius and the droplet height. Inclusion of the effect of the ionic association into the 1D model shows similar behaviors on the FSCV (Figures S18 and S19, SI). Better agreement with the experimental data is expected by fine-tuning of the standard rate constants and charge transfer coefficients of the ion and electron transfer reactions.

The effect of the standard rate constants for both ion transfer and electron transfer reactions were investigated, as shown in Figures S12 and S13 in SI. With $r_{\mathrm{d}}=2 \mu \mathrm{m}$ and $h=1.4 r_{\mathrm{d}}$ the simulated FSCV is more sensitive to the electron-transfer parameters: decreasing the standard rate constant results in decreasing peak current and wider peak separation. Decreasing the standard rate constant for the ion transfer reaction does not significantly affect the peak current but increases the peak separation, especially at low values of $k_{\mathrm{IT}}^{0}<0.01 \mathrm{~cm} \mathrm{~s}^{-1}$. Modifying the charge transfer coefficient of the ion transfer reaction did not show any effects (data not shown), while oxidation peak currents decreased and reduction peak currents increased for low values of $\alpha_{\mathrm{ET}}$ (Figure S14 in SI). Further discussion is available in the Section 5 in the SI. Note that an additional peak appears at ca. $1100 \mathrm{mV}$ in the forward scan in 
panel "I" of Figure 2A, and the current increases exponentially at an even higher potential limit in Figure 2B. This might be caused by onset of oxidation of trace impurities in the IL-PA $(\geq 97 \%)$ in toluene and oxidation of water or IL-PA, respectively. Further investigation is required to understand this process in detail and will be addressed in our future work.

Emulsion Droplets Collision Mechanism. The i-t profile of the FSCV in panel "II" of Figure 2A shows that current decreases after some cycles. As both ET and IT reactions are reversible, and nothing is consumed in the reaction, FSCV should reach a steady-state and stay there indefinitely. The gradually decreased current response might be due to three reasons: (1) something decomposes and blocks the electrode surface; (2) the droplet detaches and moves into the bulk; or (3) the droplet moves away from the middle part of the UME surface toward the insulating sheath. Because multiple collisions are observed (Figure 1), electrode fouling seems unlikely. Based on simulations (Figure 3), the droplet will initially wet the electrode surface reasonably well, with $h \approx 1$ to $1.5 r_{\mathrm{d}}$. However, after some potential cycles, the droplet will move away from the UME surface, with increasing $h$ values, and will finally leave completely. This scenario has been observed previously. ${ }^{51}$ It is also very likely that the droplet will move from the center of the UME toward the insulating sheath due to the edge effect of the UME. ${ }^{71}$ Note that some parts of the droplet may remain in contact with the electrochemically active UME surface, as being evidenced from the residual current at $535 \mathrm{~s}$ in Figure 2A II.

TBAAc concentration effect including association and/or precipitation on the FSCV was investigated by both experiments and simulations, as detailed in Section 6 of the SI. Association and precipitation between $\mathrm{Rb}^{+}$and $\mathrm{Ac}^{-}$could partially explain the discrepancy between the experimental CV and the simulated one in Figure 2D. As the ion transfer potentials of $\mathrm{ClO}_{4}^{-}$and $\mathrm{Ac}^{-}$could not be distinguished from each other in experimental FSCV (Figure 2B), we applied second-order harmonic frequency domain analysis of FSCV to investigate the collision response (Section 7 in SI). The nonFaradaic background component of voltammogram was linear in nature and mainly distributed in the fundamental excitation frequency and odd harmonics, ${ }^{55}$ and thus, the exploitation of the second-order harmonic was justified, to obtain a higher signal-to-noise $(\mathrm{S} / \mathrm{N})$ ratio. $^{72}$ This was corroborated by the observations of $\mathrm{ClO}_{4}{ }^{-}$and $\mathrm{Ac}^{-}$transfer presented herein, which showed clear differentiation in the phase angles of the secondorder harmonic analysis (Figure S20 in SI). The results above illustrated the impressive detection selectivity and sensitivity of harmonic voltammetry in the frequency domain.

Besides studying ion transfer, the interaction between the droplet and the UME during collision was also investigated by FTSV experiments in conjunction with FSCVs (Figure 2) and FE simulations (Section 5 in SI). In our previous work, we have summarized four collision modes, which are differentiated by their phase angle. ${ }^{51}$ Here we revealed some new interaction behaviors (cases II, III, and IV in Figure 5) by a comprehensive analysis of phase angle, current evolution in multiple CVs, and the FE simulations. In case I of Figure $5 \mathrm{~A}$ where $5 \mathrm{mM}$ $\mathrm{TBAClO}_{4}$ is in aqueous solution, a toluene droplet with trapped rubrene collides with the UME surface directly and then "ET coupled IT" occurs. The current suddenly increases and then slightly decreases to a quasi-constant level shown in the first row of "Response" column, due to the fast steady-state diffusion of $\mathrm{Rb}$ within the tiny droplet. This Faradaic process is

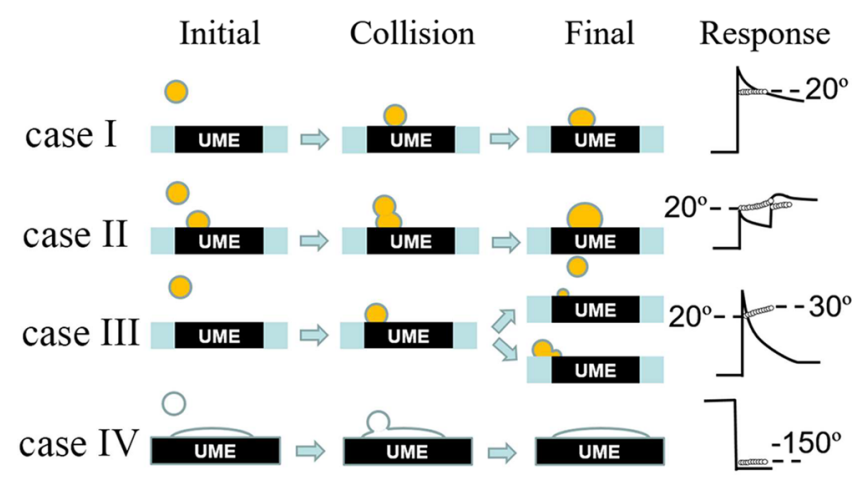

Figure 5. Four collision modes revealed by FTSV. Under the condition of $5 \mathrm{mM} \mathrm{TBAClO}_{4}$ in aqueous phase, (I) A toluene droplet containing $5 \mathrm{mM}$ rubrene collided with the UME surface directly and then attached to it after rubrene electrolysis. (II) A toluene droplet containing $5 \mathrm{mM}$ rubrene collided with another attached droplet/film formed by preceding collisions, and then fused with the droplet/film. (III) A toluene droplet containing $5 \mathrm{mM}$ rubrene collided with the UME and then either detached gradually from the UME or moved away from the UME center to the edge, resulting in the partial contact between the droplet and the glass sheath. (IV) A toluene droplet without contents (no rubrene) collided with the toluene film, and then fuses with the film. The first-order harmonic magnitude (-) after background subtraction, and the phase angle $(O)$ induced by the collision events of the four cases are shown in the "Response" column.

characterized with a phase angle of $20^{\circ}$. Case II illustrates a toluene droplet colliding onto another oil droplet/film formed by preceding collisions and then fusing with the droplet/film. This collision mechanism is implied by the second current step with the same phase angle of $20^{\circ}$ superimposed onto the first current step, as depicted in the second row of "Response" column. Case III was revealed by the gradual decrease of the current with multiple potential scans in panel "II" of Figure 2A and FE simulations (Figure 3): the redox-active droplet first collides with the UME surface and then either detaches gradually from the UME or moves away from the UME center to the edge, resulting in partial contact between the droplet and the glass sheath. The latter was implied by the residual current remaining at $535 \mathrm{~s}$ in Figure 2A II, as has been discussed before. The faster current decay and the gradual change of phase angle from $20^{\circ}$ to $30^{\circ}$ in the third row of "Response" column further corroborate these behaviors. Interestingly, we also observed a response characterized by a sudden stepwise current decrease, inducing a phase angle of about $-150^{\circ}$. This scenario is visualized in case IV in Figure 5. It occurs when a toluene droplet without the redox probe collides with the oil droplet/ film. This phenomenon is confirmed by another independent experiment shown in Figure S21 in SI. The drastic difference in contact angle between cases I-III and IV is derived from the different collision stages: early stage for case I-III and later stage for case IV where a toluene film has been formed by preceding collisions. ${ }^{51}$ The frequency/time domain analyses in combination with FE simulations have shed new light on the collision mechanism of emulsion droplets.

\section{CONCLUSIONS}

In conclusion, FSCV/FTSV, characterized with high temporal and potential resolution, has been successfully applied in conjunction with the FE simulations to study single-emulsion droplet collision electrochemistry with the process of "ET coupled IT". The thermodynamics of ion transfer (i.e., 
hydrophilicity/lipophilicity of ions) at the soft interface between water and toluene of very low dielectric constant has been estimated via single-emulsion droplet collision electrochemistry. Emulsion droplet size and shape distributions upon collision have also been uncovered by comparison between experiments and simulations. This cannot be addressed easily using other methods and extends earlier works using sampled current voltammetry, which requires additional experimental time and has the risk of potential drift. ${ }^{32}$ Some new physical insights were also revealed herein: for example, it is the $i R$ drop in the aqueous phase where few dissociated ions are present for the salts of $\mathrm{TEA}^{+}$, or $\mathrm{TBA}^{+}$, or $\mathrm{THA}^{+}$with their common anion $\mathrm{PF}_{6}{ }^{-}$, leading to a larger peak-to-peak separation than that for $\mathrm{TBAClO}_{4}, \mathrm{TBAAc}$, and $\mathrm{TMAPF}_{6}$. Finally, the mechanisms with three new collision modes have been investigated in detail by applying time/frequency-domain analysis and FE FSCV simulations. Herein, the methodology of "Single Organic Droplet Collision Voltammogram via Electron Transfer Coupled Ion Transfer" has been established and which can be applied in the future to a broader range of complicated (electro)chemical problems such as phase transfer catalysis in organic chemistry, colloidal and nano science, ion sensors, microfluidics, biochemical processes like vesicle-based drug delivery and exocytosis, and so forth.

\section{ASSOCIATED CONTENT}

\section{S Supporting Information}

The Supporting Information is available free of charge on the ACS Publications website at DOI: 10.1021/acs.analchem.7b02072.

Emulsion size distributions by DLS, thermodynamic calculations for ionic phase distribution, peak current distributions and analysis, collision frequency analysis, finite element simulations, TBAAc concentration effect, and phase angle analysis and collision mechanisms (PDF)

\section{AUTHOR INFORMATION}

\section{Corresponding Authors}

*E-mail for L.S.W.: wanglsh@scut.edu.cn.

*E-mails for H.Q.D.: haiqiang.deng@mail.huji.ac.il or hqdeng2010@gmail.com.

\section{ORCID}

Pekka Peljo: 0000-0002-1229-2261

Lishi Wang: 0000-0002-7700-7847

Author Contributions

II (C.L., P.P.) Equal contribution.

\section{Notes}

The authors declare no competing financial interest.

\section{ACKNOWLEDGMENTS}

We gratefully acknowledge the financial support from the National Natural Science Foundation of China (Nos. 21475046, 21427809, 21605051) and the Fundamental Research Funds for the Central Universities (No. 2015ZP028). H.Q.D. acknowledges the financial support from the PBC Fellowship jointly sponsored by the Planning and Budgeting Committee of the Council for Higher Education in Israel and the Hebrew University of Jerusalem and also appreciates Prof. Daniel Mandler for hosting his postdoctoral studies. P.P. acknowledges the financial support from the Swiss
National Science Foundation, grant Ambizione Energy 160553. The authors also acknowledge Dr. Christopher R. Dennison (LEPA, EPFL, Switzerland) for improving the English of the paper.

\section{REFERENCES}

(1) Blank, M.; Feig, S. Science 1963, 141, 1173-1174.

(2) Mitchell, P. Nature 1961, 191, 144-148.

(3) Benjamin, I. J. Phys. Chem. B 2013, 117, 4325-4331.

(4) Marcus, Y.; Kolarik, Z. J. Chem. Eng. Data 1973, 18, 155-163.

(5) Stockmann, T. J.; Ding, Z. Anal. Chem. 2011, 83, 7542-7549.

(6) Liu, B.; Mirkin, M. V. Anal. Chem. 2001, 73, 670A-677 A.

(7) Samec, Z. Pure Appl. Chem. 2004, 76, 2147-2180.

(8) Girault, H. H. In Electroanalytical Chemistry: A Series of Advances; Bard, A. J., Zoski, C. G., Eds.; CRC Press: Boca Raton, FL, 2010; Vol. 23, pp 1-104.

(9) Liu, S.; Li, Q.; Shao, Y. Chem. Soc. Rev. 2011, 40, 2236-2253.

(10) Peljo, P.; Girault, H. H. In Encyclopedia of Analytical Chemistry; Meyers, R. A., Ed.; John Wiley \& Sons, Ltd: Hoboken, NJ, 2012.

(11) Gavach, C.; Henry, F. J. Electroanal. Chem. Interfacial Electrochem. 1974, 54, 361-370.

(12) Samec, Z.; Mareček, V.; Koryta, J.; Khalil, M. W. J. Electroanal. Chem. Interfacial Electrochem. 1977, 83, 393-397.

(13) Peljo, P.; Murtomäki, L.; Kallio, T.; Xu, H. J.; Meyer, M.; Gros, C. P.; Barbe, J. M.; Girault, H. H.; Laasonen, K.; Kontturi, K. J. Am. Chem. Soc. 2012, 134, 5974-5984.

(14) Shi, C.; Anson, F. C. Anal. Chem. 1998, 70, 3114-3118.

(15) Quentel, F.; Mirčeski, V.; L'Her, M. Anal. Chem. 2005, 77, 1940-1949.

(16) Deng, H.; Huang, X.; Wang, L.; Tang, A. Electrochem. Commun. 2009, 11, 1333-1336.

(17) Deng, H.; Huang, X.; Wang, L. Langmuir 2010, 26, 1920919216.

(18) Taylor, G.; Girault, H. H. J. Electroanal. Chem. Interfacial Electrochem. 1986, 208, 179-183.

(19) Jing, P.; Zhang, M.; Hu, H.; Xu, X.; Liang, Z.; Li, B.; Shen, L.; Xie, S.; Pereira, C. M.; Shao, Y. Angew. Chem., Int. Ed. 2006, 45, 68616864.

(20) Rodgers, P. J.; Amemiya, S. Anal. Chem. 2007, 79, 9276-9285.

(21) Stockmann, T. J.; Ding, Z. J. Electroanal. Chem. 2010, 649, 2331.

(22) Wang, Y.; Kakiuchi, T.; Yasui, Y.; Mirkin, M. V. J. Am. Chem. Soc. 2010, 132, 16945-16952.

(23) Stockmann, T. J.; Lu, Y.; Zhang, J.; Girault, H. H.; Ding, Z. Chem. - Eur. J. 2011, 17, 13206-13216.

(24) Stockmann, T. J.; Guterman, R.; Ragogna, P. J.; Ding, Z. Langmuir 2016, 32, 12966-12974.

(25) Campbell, J. A.; Girault, H. H. J. Electroanal. Chem. Interfacial Electrochem. 1989, 266, 465-469.

(26) Arrigan, D. W. M.; Liu, Y. Annu. Rev. Anal. Chem. 2016, 9, 145161.

(27) Deng, H.; Peljo, P.; Momotenko, D.; Cortés-Salazar, F.; Jane Stockmann, T.; Kontturi, K.; Opallo, M.; Girault, H. H. J. Electroanal. Chem. 2014, 732, 101-109.

(28) Li, F.; Su, B.; Salazar, F. C.; Nia, R. P.; Girault, H. H. Electrochem. Commun. 2009, 11, 473-476.

(29) Li, F.; Unwin, P. R. J. Phys. Chem. C 2015, 119, 4031-4043.

(30) Sun, P.; Zhang, Z.; Gao, Z.; Shao, Y. Angew. Chem., Int. Ed. 2002, 41, 3445-3448.

(31) Wei, C.; Bard, A. J.; Mirkin, M. V. J. Phys. Chem. 1995, 99, 16033-16042.

(32) Deng, H.; Dick, J. E.; Kummer, S.; Kragl, U.; Strauss, S. H.; Bard, A. J. Anal. Chem. 2016, 88, 7754-7761.

(33) Cheng, W.; Compton, R. G. TrAC, Trends Anal. Chem. 2014, 58, 79-89.

(34) Kleijn, S. E. F.; Lai, S. C. S.; Koper, M. T. M.; Unwin, P. R. Angew. Chem., Int. Ed. 2014, 53, 3558-3586. 
(35) Bard, A.; Boika, A.; Kwon, S.; Park, J.; Thorgaard, S. In Nanoelectrochemistry; Mirkin, M. V., Amemiya, S., Eds.; CRC Press: Boca Raton, FL, 2015; pp 241-292.

(36) Scanlon, M. D.; Peljo, P.; Mendez, M. A.; Smirnov, E.; Girault, H. H. Chem. Sci. 2015, 6, 2705-2720.

(37) Anderson, T. J.; Zhang, B. Acc. Chem. Res. 2016, 49, 2625-2631. (38) Kim, J.; Dick, J. E.; Bard, A. J. Acc. Chem. Res. 2016, 49, 25872595.

(39) Oja, S. M.; Fan, Y.; Armstrong, C. M.; Defnet, P.; Zhang, B. Anal. Chem. 2016, 88, 414-430.

(40) Peng, Y.-Y.; Qian, R.-C.; Hafez, M. E.; Long, Y.-T. ChemElectroChem 2017, 4, 977-985.

(41) Sokolov, S. V.; Eloul, S.; Katelhon, E.; Batchelor-McAuley, C.; Compton, R. G. Phys. Chem. Chem. Phys. 2017, 19, 28-43.

(42) Ying, Y.-L.; Ding, Z.; Zhan, D.; Long, Y.-T. Chem. Sci. 2017, 8, $3338-3348$.

(43) Quinn, B. M.; van't Hof, P. G.; Lemay, S. G. J. Am. Chem. Soc. 2004, 126, 8360-8361.

(44) Kim, B.-K.; Boika, A.; Kim, J.; Dick, J. E.; Bard, A. J. J. Am. Chem. Soc. 2014, 136, 4849-4852.

(45) Zhou, Y.-G.; Rees, N. V.; Compton, R. G. Angew. Chem., Int. Ed. 2011, 50, 4219-4221.

(46) Xiao, X.; Bard, A. J. J. Am. Chem. Soc. 2007, 129, 9610-9612. (47) Xiao, X.; Fan, F.-R. F.; Zhou, J.; Bard, A. J. J. Am. Chem. Soc. 2008, 130, 16669-16677.

(48) Kleijn, S. E. F.; Lai, S. C. S.; Miller, T. S.; Yanson, A. I.; Koper, M. T. M.; Unwin, P. R. J. Am. Chem. Soc. 2012, 134, 18558-18561.

(49) Dasari, R.; Robinson, D. A.; Stevenson, K. J. J. Am. Chem. Soc. 2013, 135, 570-573.

(50) Guo, Z.; Percival, S. J.; Zhang, B. J. Am. Chem. Soc. 2014, 136, $8879-8882$.

(51) Huang, X.; Deng, H.; Liu, C.; Jiang, J.; Zeng, Q.; Wang, L. Chem. - Eur. J. 2016, 22, 9523-9527.

(52) Svetličić, V.; Ivošević, N.; Kovač, S.; Žutić, V. Langmuir 2000, 16, 8217-8220.

(53) Percival, S. J.; Zhang, B. J. Phys. Chem. C 2016, 120, 2053620546.

(54) Liu, C.; Cheng, W.; Zeng, Q.; Huang, X.; Wang, L. J. Electroanal. Chem. 2017, 784, 145-152.

(55) Singhal, P.; Kawagoe, K. T.; Christian, C. N.; Kuhr, W. G. Anal. Chem. 1997, 69, 1662-1668.

(56) Fang, Y.; Huang, X.; Wang, L. Anal. Chem. 2015, 87, 448-456.

(57) Batchelor-McAuley, C.; Little, C. A.; Sokolov, S. V.; Kätelhön, E.; Zampardi, G.; Compton, R. G. Anal. Chem. 2016, 88, 1121311221.

(58) Li, X.; Batchelor-McAuley, C.; Whitby, S. A. I.; Tschulik, K.; Shao, L.; Compton, R. G. Angew. Chem., Int. Ed. 2016, 55, 4296-4299.

(59) Kasuno, M.; Matsuyama, Y.; Iijima, M. ChemElectroChem 2016, 3, 694-697.

(60) Amatore, C.; Arbault, S.; Guille, M.; Lemaittre, F. Chem. Rev. 2008, 108, 2585-2621.

(61) Cans, A.-S.; Ewing, A. G. J. Solid State Electrochem. 2011, 15, $1437-1450$.

(62) Cheng, W.; Compton, R. G. Angew. Chem., Int. Ed. 2014, 53, 13928-13930.

(63) Dunevall, J.; Fathali, H.; Najafinobar, N.; Lovric, J.; Wigström, J.; Cans, A.-S.; Ewing, A. G. J. Am. Chem. Soc. 2015, 137, 4344-4346.

(64) Gao, F.; Yang, Y.; Liu, J.; Shao, H. Ionics 2010, 16, 45-50.

(65) Bowen, E. J.; Steadman, F. J. Chem. Soc. 1934, 1098-1101.

(66) Johans, C.; Behrens, M. A.; Bergquist, K. E.; Olsson, U.; Manzanares, J. A. Langmuir 2013, 29, 15738-15746.

(67) Zhou, M.; Gan, S.; Zhong, L.; Su, B.; Niu, L. Anal. Chem. 2010, $82,7857-7860$.

(68) Koczorowski, Z. In Liquid Interfaces In Chemical, Biological And Pharmaceutical Applications; Volkov, A. G., Ed.; CRC Press: Boca Raton, FL, 2001.

(69) Hubbard, A. T. J. Electroanal. Chem. Interfacial Electrochem. 1969, 22, 165-174.

(70) Tom, G. M.; Hubbard, A. T. Anal. Chem. 1971, 43, 671-674.
(71) Fosdick, S. E.; Anderson, M. J.; Nettleton, E. G.; Crooks, R. M. J. Am. Chem. Soc. 2013, 135, 5994-5997.

(72) Chen, W.; Wang, L.; Huang, X.; Wang, M. Anal. Chem. 2013, $85,83-90$. 